\title{
Recent Developments in Prostate Cancer Screening Guidelines
}

\author{
Ilija Aleksic ${ }^{1}$, Vladamir Mouraviev ${ }^{2 *}$ and David Albala ${ }^{3}$
}

${ }^{1}$ SUNY Upstate Medical University, USA

${ }^{2}$ Research Director, Associated Medical Professionals of New York, USA

${ }^{3}$ Medical Director, Associated Medical Professionals of New York, USA

\begin{abstract}
Screening recommendations must take into consideration the variable course of $\mathrm{PCa}$ for individual patients. While prostate cancer is commonly referred to as a slow growing process, this over-generalization is filled with potentially dangerous assumptions for individuals who harbor aggressive forms of disease. Widespread screening with prostate specific antigen began in the late 1980s, with mortality due to prostate cancer in decline for the past two decades. This approach has led to a substantial decrease in the diagnosis of high-grade disease. Therefore, population-wide data supports the utility of prostate cancer screening with prostate specific antigen.
\end{abstract}

The purpose of this article is to evaluate the current status of prostate screening in light of the US Preventative Service Task Force guidelines against routine screening and the more recently published American Urological Association guidelines for shared decision-making centered on patient education and autonomy. The difference in recommendations arises from the interpretation of the results from large, randomized prospective screening trials. While the studies may not illustrate a significant benefit for screening, they have notable limitations that make definitive conclusions based on their results, difficult to derive.

Epidemiologic data supports the use of screening modalities for diseases with high prevalence and a prolonged asymptomatic phase. As with any test, there are risks associated with prostate specific antigen screening. The major concerns are over diagnosis and over treatment. While the scientific community works toward more specific screening tests, it is imperative that individual patients are educated and involved in the shared decision-making. Patient perception of their individual harm and benefit becomes a major contributor to the decision-making process when evidence is largely inconclusive and unable to provide definitive direction for the practitioner.

Keywords: Prostate cancer; Prostate specific antigen; Screening; Guidelines

\section{Introduction}

The continued study and implementation of screening modalities for prostate cancer ( $\mathrm{PCa}$ ) is imperative because it is a leading cause of cancer death in men, second only to lung cancer. An estimated one in six men will be diagnosed with PCa during their lifetime. It is projected that 238,590 new cases will be diagnosed in 2013, resulting in 29,720 deaths $[1,2]$. At present, the most commonly used tools for detection of PCa are the Prostate Specific Antigen (PSA) test and the digital rectal exam (DRE). Together, these complementary tests provide physicians with indication of whether or not to proceed with biopsy for diagnostic confirmation. At present, the literature provides limited data on which to provide certainty for the harm or benefit of PSA-based prostate screening. While PCa is commonly referred to as a slow growing disease, this over-generalization is filled with potentially dangerous assumptions for individuals who harbor aggressive forms of disease. Therefore, screening guidelines must take into consideration the variable course of PCa for individual patients.

PSA was initially adopted as a method to monitor PCa progression; however, beginning in the late 1980s, it was widely accepted as a means to screen for PCa. From the induction of PSA screening, a $30 \%$ decline in overall mortality secondary to PCa has been observed [3]. Epidemiologic models have illustrated that up to $70 \%$ of the mortality decrease may be attributed to screening alone [4], while the remainder may be attributed to improved management of the cancer itself. A recent National Institutes of Health Consensus Development Conference concluded that prior to PSA screening, PCa was detected due to a positive DRE alone or presence of symptoms, both indicting an already high grade, and often lethal, stage of the disease [5]. The screening practices of the past two decades have resulted in an increase in diagnosis of localized PCa with a subsequent decrease in diagnosis of high-grade disease.

The purpose of this article is to evaluate the current status of prostate screening, especially in light of the definitive US Preventative Service Task Force (USPSTF) decision against routine screening and the recently published American Urological Association (AUA) guidelines for shared and informed decision making. Recommendations commonly center on a consensus that screening decisions need to be based on an informed patient-practitioner discussion. Despite this accepted practice, in 2012 the USPSTF published a recommendation against all PSA screening, regardless of age, race, or family history [6] On the contrary, in 2013, the AUA updated its guidelines to improve the benefit-to-harm ratio that has been under attack in recent years [7]. This is accomplished by recommending increased intervals for screening while making decisions centered on patient education and preference. They indicate that ample evidence is available to support

*Corresponding author: Vladamir Mouraviev, Research Director, Associated Medical Professionals of New York, A.M.P. Urology, 1226 East Water Street, Syracuse, NY 13210, USA, Tel: (315) 478-4185; Fax: (315) 478-0840; E-mail: vmouraviev@ampofny.com

Received May 06, 2013; Accepted May 22, 2013; Published May 24, 2013

Citation: Aleksic I, Mouraviev V, Albala D (2013) Recent Developments in Prostate Cancer Screening Guidelines. J Cancer Sci Ther S7: 005. doi:10.4172/1948-5956. S7-005

Copyright: (C) 2013 Aleksic I, et al. This is an open-access article distributed under the terms of the Creative Commons Attribution License, which permits unrestricted use, distribution, and reproduction in any medium, provided the original author and source are credited. 
utilization of a shared decision-making strategy that increases patient knowledge and promotes greater involvement in making decisions.

The USPTSF recommendations are updated about every five years, unless the scientific environment urges an early revision. These new proposed guidelines were put forth only four years after the last recommendation; however, they are based on studies with notable limitations. The Task Force concluded that the harms of screening for PCa outweigh the benefits. Their recommendation removes the purpose of an informed discussion about screening, thereby removing patient autonomy to make informed decisions about cancer screening. The AUA, on the other hand, guides the patient-practitioner conversation toward an open discussion on the risks and benefits of screening for PCa.

\section{Reasons Behind the USPSTF Recommendation}

The background for the latest USPSTF PCa recommendation for abandonment of routine PSA screening for PCa is based on two, large randomized screening trials. The two landmark trials that provided the data for the recommendation were the U.S. Prostate, Lung, Colorectal, and Ovarian (PLCO) cancer screening trial and the European Randomized Study of Screening for Prostate Cancer (ERSPC), which showed non-significant or no mortality benefit with PSA screening [8]. The results of the two trials vary, where PLCO and ERPC show a relative reduction in mortality (with $95 \%$ confidence interval) of 1.09 $(0.87-1.36)$ and $0.79(0.68-0.91)$ respectively $[9,10]$. However, these studies provide more questions than answers while identifying clear gaps in the evidence base. Notably, only an estimated ten-year followup was cited. Also, data in regards to men outside of the age groups enrolled in the studies is virtually non-existent. Most importantly, key outcomes such as over diagnosis and the additional number needed to treat cannot be readily deduced from data generated by the trials [7]. It is clearly evident that definitive conclusions were made with incomplete evidence [11] with significant limitations. Nevertheless, the Task Force concluded that the vast majority of men who were screened and received PCa treatment would have experienced the same longevity and quality of life regardless of intervention.

The USPSTF argued that the trend of decreased PCa mortality was not due to PSA-driven early detection. However, the studies cited do not provide us with another explanation for the decline in mortality. Multiple concerns about the inherent limitations for the screening studies used have been raised. The major concern for the studies is the short-term follow up of about ten years. Even in the limited understanding of PCa biology and its phenotypic variability, the slow course of the disease process is agreed upon. Therefore, making any conclusions without adequate follow-up involves numerous assumptions and extrapolations. Additional limitations of the studies include contamination by opportunistic PSA screenings of subjects in the control arm, which was reported in detail [12]. The contamination rate was estimated at 25\% in the ERSPC trial and 77\% [13] in the PLCO trial [14], with substantial prescreening prior to trial initiation.

Further limitations include an oversight toward higher complication rates for procedures in advanced disease, which will increase in incidence as a result of lack of population screening. Furthermore, quality of life issues for advanced disease such as bone pain, pathologic fractures, and urinary tract obstructions were entirely overlooked by the analysis of the data [15]. On the other hand, the results of another screening trial, the Goteborg Center randomized screening trial, demonstrated that PCa mortality was reduced by nearly half after 14 years of follow-up [16]. Therefore, possibly under increased pressure due to the world economic recession, the Task Force arrived to a premature conclusion that population-wide screening is deleterious for the U.S. health-care system and it should never be recommended.

\section{Shortcomings of PCa Screening}

Criticism for the use of PSA as a screening modality is that it leads to over diagnosis and over treatment, with resulting adverse effects for a disease process that may not have caused any harm without treatment. The major argument against PSA screening is the prevalence of over treatment for low-risk PCa. Up to $94 \%$ of all men with lowgrade PCa continue to receive radical treatment in the U.S. [17]. Given our knowledge of the biology of PCa, it is highly unlikely that nearly all diagnosed PCa requires such treatment. Complications of radical treatment include incontinence, impotence and death. Even though complications are rare, they are an unnecessary risk for the patient who has low risk disease with no benefit from treatment.

For complications of the biopsy itself, by using estimates of the number of prostate biopsies per year and the annual incidence of $\mathrm{PCa}$, Vickers et al. estimated that PSA testing leads to approximately 750,000 unnecessary biopsies for each year in the United States [18]. The number of biopsies is significant because life-threatening complications such as urosepsis are possible. Also concerning are the less serious complications such as pain, localized infection and psychological effects of a false positive test results. However, in regards to PSA-anxiety, two randomized PSA screening trials reported no detrimental effect to anxiety levels due to screening $[19,20]$. Nevertheless, risks of a biopsy are themselves a reason for developing novel methods of discerning the patients who need such invasive work-up.

The treatment paradigm for managing localized PCa in an effort to minimize over-treatment, is to distinguish patients with aggressive cancer that require aggressive therapy from those who may benefit from either an organ-sparing approach or those who likely do not need any intervention at the time of diagnosis. It is imperative for practitioner and patient to know that not all men who are screened need to pursue a biopsy and not all men diagnosed require treatment.

\section{The Response to the USPSTF Guideline}

The American Urological Association (AUA) replied immediately when ad-hoc panel of physicians that criticized the USPSTF as "a panel that does not include urologists or cancer specialists," underestimating the benefits and overestimating the harms of PCa screening [15]. The experts from the panel stressed that the Task Force has gravely misinterpreted the natural history of PCa.

Crawford et al. estimated the clinical and economic effects of increasing the current one year PSA screening interval by one to four years based on the PLCO Cancer Screening Trial results [21]. They found significant financial savings, particularly for a 5-year screening interval, with estimated national savings of $\$ 3.9$ billion. However, taking into consideration the amount of potentially missed cancers, the authors concluded that a "no screening" policy would be a poor healthcost investment.

The USPSTF has put itself in a conundrum with probable societal consequences if the landscape changes away from the detection of a prevalent disease at an early stage and toward detection at an advanced stage. For instance, President Obama himself had a screening PSA test as part of his annual physical at Walter Reed earlier in 2012. 
Presumably, he was counseled on the benefits and risks of the test, and chose to be screened. It would be a societal loss if that choice, and with it patient autonomy, were taken away from other men at similar risk. Although a no-screening policy would reduce screening health expenditure in the short term, it would be a poor investment because cost of PCa management may not change after taking into account the cost of treatment of advanced disease and the savings made possible by implementing variable interval screening.

Dr. Moul highlighted another major limitation of the Task Force approach, which missed the nuanced potential of PSA as a riskassessment and stratification tool [21]. For instance, if a 40 year-old man has a baseline PSA of less than $1 \mathrm{ng} / \mathrm{mL}$, that may indicate if PCa were to occur in the future, it would be low-risk. On the contrary, a 40 -year-old with PSA greater than $1.5-2.5 \mathrm{ng} / \mathrm{mL}$ has to be followed up more closely. Data from the afore-mentioned trials reviewed by USPSTF represent too narrow of an approach for PSA values. This limitation raised many concerns about unintended consequences of one size fits all approach to discouraging all PSA screening.

Several guidelines have been published since the dramatic display by USPSTF. The AUA commissioned an independent panel to conduct a systematic review on the use of the PSA test for early detection of PCa. In April of 2013, the AUA Board of Directors approved the following guidelines. These include no routine screening between the age of 40 and 54, with individualized decisions for higher risk individuals. For men ages 55 to 69 , they recommend shared decision making in considering PSA screening, and proceeding based on a patient preference, with screening for an interval of two years or more. It is expected that increased interval time preserves screening benefit while minimizing the risks of false positives, over-diagnosis and over-treatment. They further suggest that intervals themselves can be individualized by a baseline PSA level. Lastly, while not recommending routine PSA screening for men over 70, they suggest that some who are in excellent health may benefit from screening [7]. The AUA guidelines derive from data interpretation through the lens of the individual, with emphasis on informative decision-making, versus the USPSTFs public health perspective.

The fundamental differences in these guidelines highlight limitations of PSA as a screening modality. However, a superior alternative has yet to become available. As basic research moves forward in the discovery of new biomarkers, the current armamentarium of tests for urine, blood, and genes must be fully exploited and new screening combinations must be attempted in order to deduce the most sensitive and specific ways to detect PCa. While research moves toward new discoveries, the utility of PSA for detecting early stages of disease must not be overlooked. The PSA question must be approached with the tools we have at hand. Foregoing PCa screening by way of available methodologies, as suggested by the USPSTF, will prove to be extremely harmful to the well being of many.

Utilization of early PSA levels as baseline is one method of stratifying patients to individual risk categories. A recent case control study in a large, screening-naïve cohort has demonstrated the utility of a single PSA measurement in the fourth decade can be used to determine the frequency of future screening [22]. This study, in combination a previous series by the same group [23] illustrated a feasible approach to risk-stratification with a single PSA measurement and subsequent frequency of screening. This approach functions to decrease unnecessary biopsies, while permitting more frequent screening for those with an increased risk.
Another strategy that that can be used in combination with PSAs are mathematical tools that use patient characteristics that have already been associated with PCa. Risk-calculators may provide the necessary guidance to risk-stratify patients in more specific categories [24]. Risk calculators may allow to further sub-classify patients not only by risk of $\mathrm{PCa}$, but also by risk of high grade PCa [25]. The ability to delineate risk for low and high grade PCa will allow for more individualized screening and treatment regimens. Providing the tools for PCa risk stratification, as well as further stratification based on PCa aggressiveness potential, will enable physicians to make sound judgments on screening intervals and types of appropriate treatment for individual patients.

\section{Lessons Learned}

The take home message from the USPSTF is that benefits rendered are not substantial enough to justify the risks of screening and treatment as well as the cost to the health-care system. At the same time, side effects such as erectile dysfunction and incontinence are treatable. Symptoms due to advanced PCa are more common, more difficult and more costly to manage than the rare complications of screening or treating low-grade disease.

The Task Force also did not consider the economic impact of treating advanced PCa versus screening for low-grade disease. Upon considering the financial burden of metastatic disease, in addition to a cost of life and quality, it would be imperative to avoid a return to the pre-PSA era for economical reasons as well. Ultimately, it is clear that policymakers must to consider the risks of the USPSTF recommendation before making reimbursement decisions that would jeopardize patient health.

It is simple to imagine an environment where Medicare and Medicaid cease to reimburse PSA screening for PCa. Whether large private insurers monopolize screening coverage or follow suit, an accentuation of an already prominent health-disparity problem is a guaranteed result. Socioeconomic status (SES), a well accepted determinant of overall health and life expectancy, will become the major risk factor for PCa mortality because degree of spread at diagnosis will coincide with income level. The national problem of access-to-care will be compounded by access-to-screening. Unfortunately, the populations most likely affected due to SES, namely men of African American, Latino or Hispanic descent, are the same populations that are most likely to have high-risk features for aggressive and life-threatening PCa. This will certainly have serious consequences that need to be identified and addressed in order to prevent a further societal decline into SES driven health outcomes.

The main lesson arising from the current debate is the importance in educating patients about risks and benefits, while facilitating shared decision-making. In a situation where the harm to benefit ratio is so close that it can be used for and against intervention, patient perception of their own potential harm or benefit must be a major component of the decision made.

\section{Discussion}

In order to alleviate the issue of over-diagnosis, a method of individualized screening regimens must be developed. A useful approach is to stratify patients by both risk for PCa and risk for advanced PCa. Risk-stratification will result in shorter screening intervals for patients at higher risk and longer intervals for patients at lower risk. A remaining concern about a move from a population wide screening program is that aggressive disease phenotypes will still 
occur in the low-risk cohort of patients. It is because of this reason that implementing guidelines based on risk will require continual update and revision.

In an effort to minimize over-treatment once a diagnosis has been made, stratification of therapy based on individual PCa characteristics, will allow for appropriate regimens to be administered. Improved guidance on individualized treatment choice for patients who are positive for PCa will further alleviate concerns about over-treatment. Patients with low-risk disease with low aggressive potential can then be appropriately informed of risks and benefits of surveillance versus treatment, and be given all treatment options including focal therapy, radial surgery, radiotherapy, as well as adjuvant therapies. In order to alleviate the issue of over-treatment after diagnosis, riskbased stratification of treatment decisions must also be developed to complement a risk-based stratification of screening.

\section{References}

1. Siegel R, Naishadham D, Jemal A (2013) Cancer statistics, 2013. CA Cancer J Clin 63: 11-30.

2. Jemal A, Siegel R, Xu J, Ward E (2010) Cancer statistics, 2010. CA Cancer J Clin 60: 277-300

3. Etzioni R, Gulati R, Falcon S, Penson DF (2008) Impact of PSA screening on the incidence of advanced stage prostate cancer in the United States: a surveillance modeling approach. Med Decis Making 28: 323-331.

4. Etzioni R, Tsodikov A, Mariotto A, Szabo A, Falcon S, et al. (2008) Quantifying the role of PSA screening in the US prostate cancer mortality decline. Cancer Causes Control 19: 175-181.

5. Ganz PA, Barry JM, Burke W, Col NF, Corso PS, et al. (2012) Nationa Institutes of Health State-of-the-Science Conference: role of active surveillance in the management of men with localized prostate cancer. Ann Intern Med 156: 591-595.

6. Moyer VA (2012) Screening for prostate cancer: U.S. Preventive Services Task Force recommendation statement. Ann Intern Med 157: 120-134.

7. Carter HB, Albertsen PC, Barry MJ, Etzioni R, Freedland SJ, et al. (2013) Early Detection of Prostate Cancer: AUA Guideline. J Urol

8. Chou R, Croswell JM, Dana T, Bougatsos C, Blazina I, et al. (2011) Screening for prostate cancer: a review of the evidence for the U.S. Preventive Services Task Force. Ann Intern Med 155: 762-771.

9. Andriole GL, Crawford ED, Grubb RL 3rd, Buys SS, Chia D, et al. (2009) Mortality results from a randomized prostate-cancer screening trial. $\mathrm{N}$ Engl J Med 360: 1310-1319.

10. Schröder FH, Hugosson J, Roobol MJ, Tammela TL, Ciatto S, et al. (2009) Screening and prostate-cancer mortality in a randomized European study. N Engl J Med 360: 1320-1328.

11. Carlsson S, Vickers AJ, Roobol M, Eastham J, Scardino P, et al. (2012) Prostate cancer screening: facts, statistics, and interpretation in response to the US Preventive Services Task Force Review. J Clin Oncol 30: 2581-2584.

12. Kerkhof M, Roobol MJ, Cuzick J, Sasieni P, Roemeling S, et al. (2010) Effect of the correction for noncompliance and contamination on the estimated reduction of metastatic prostate cancer within a randomized screening trial (ERSPC section Rotterdam). Int J Cancer 127: 2639-2644.

13. Schröder FH, Hugosson J, Roobol MJ, Tammela TL, Ciatto S, et al. (2012) Prostate-cancer mortality at 11 years of follow-up. N Engl J Med 366: 981-990.
14. Pinsky PF, Blacka A, Kramer BS, Miller A, Prorok PC, et al. (2010) Assessing contamination and compliance in the prostate component of the Prostate, Lung, Colorectal, and Ovarian (PLCO) Cancer Screening Trial. Clin Trials 7: 303-311.

15. Catalona WJ, D'Amico AV, Fitzgibbons WF, Kosoko-Lasaki O, Leslie SW, et al. (2012) What the U.S. Preventive Services Task Force missed in its prostate cancer screening recommendation. Ann Intern Med 157: 137-138.

16. Hugosson J, Carlsson S, Aus G, Bergdahl S, Khatami A, et al. (2010) Mortality results from the Göteborg randomised population-based prostate-cancer screening trial. Lancet Oncol 11: 725-732.

17. Cooperberg MR, Lubeck DP, Meng MV, Mehta SS, Carroll PR (2004) The changing face of low-risk prostate cancer: trends in clinical presentation and primary management. J Clin Oncol 22: 2141-2149.

18. Vickers A, Cronin A, Roobol M, Savage C, Peltola M, et al. (2010) Reducing unnecessary biopsy during prostate cancer screening using a four-kallikrein panel: an independent replication. J Clin Oncol 28: 2493-2498.

19. Macefield RC, Lane JA, Metcalfe C, Down L, Neal DE, et al. (2009) Do the risk factors of age, family history of prostate cancer or a higher prostate specific antigen level raise anxiety at prostate biopsy? Eur J Cancer 45: 2569-2573.

20. Carlsson S, Aus G, Wessman C, Hugosson J (2007) Anxiety associated with prostate cancer screening with special reference to men with a positive screening test (elevated PSA) - Results from a prospective, population-based, randomised study. Eur J Cancer 43: 2109-2116.

21. Piana R (2012) New PSA Recommendations: The Debate over Prostate Cancer Screening Continues. The ASCO POST 3.

22. Vickers AJ, Ulmert D, Sjoberg DD, Bennette CJ, Björk T, et al. (2013) Strategy for detection of prostate cancer based on relation between prostate specific antigen at age 40-55 and long term risk of metastasis: case-control study. BM 346: f2023.

23. Vickers AJ, Cronin AM, Björk T, Manjer J, Nilsson PM, et al. (2010) Prostate specific antigen concentration at age 60 and death or metastasis from prostate cancer: case-control study. BMJ 341: c4521.

24. Zhu X, Albertsen PC, Andriole GL, Roobol MJ, Schröder FH, et al. (2012) Riskbased prostate cancer screening. Eur Urol 61: 652-661.

25. Thompson IM, Ankerst DP (2012) The benefits of risk assessment tools for prostate cancer. Eur Urol 61: 662-663.
This article was originally published in a special issue, Cancer Screening \& Early Detection handled by Editor(s). Dr. Arutselvan Natarajan, Stanford University, United States 